\title{
Diseño y puesta en marcha de un proyecto de Aprendizaje y Servicio en Análisis Económico de las Organizaciones
}

\author{
García-Álvarez, María Teresa; Teijeiro-Álvarez, Mercedes \\ Universidade da Coruña, Facultad de Economía y Empresa
}

\section{RESUMEN}

El presente trabajo desarrolla el diseño y puesta en marcha de un Proyecto de Aprendizaje y Servicio en la asignatura Análisis Económico de las Organizaciones, asignatura troncal del doble grado de Administración y Dirección de Empresas-Derecho de la Universidade da Coruña. El objetivo de aplicar esta metodología es combinar procesos de aprendizaje y de servicio comunitario que promuevan del desarrollo de actividades estudiantiles solidarias mientras los alumnos adquieren las competencias de la asignatura previamente programadas en la planificación docente. Asimismo, este proyecto ha sido integrado dentro de la plataforma virtual Moodle, con la finalidad de utilizar tecnologías de información y comunicación que permitan el aprendizaje cooperativo en su desarrollo.

Los resultados muestran que el proyecto Aprendizaje y Servicio ha permitido que los estudiantes adquirieran las competencias programadas en la planificación docente, tanto las relacionadas con la organización y gestión de empresas, como las relacionadas con su compromiso con la comunidad. Asimismo, la entidad colaboradora y el profesorado han valorado de forma positiva el desarrollo de dicho proyecto, mostrando su interés en mantener colaboraciones futuras.

PALABRAS CLAVE: Proyecto Aprendizaje y Servicio, Tecnologías de la Información y Comunicación, Análisis Económico de las Organizaciones. 


\section{CITA RECOMENDADA:}

García-Álvarez, M. T., Teijeiro-Álvarez, M. (2018). Diseño y puesta en marcha de un proyecto de Aprendizaje y Servicio en Análisis Económico de las Organizaciones. En E. de la Torre Fernández (ed.) (2018). Contextos universitarios transformadores: retos e ideas innovadoras. II Xornadas de Innovación Docente. Cufie. Universidade da Coruña (pág. 169-182).

DOI capítulo: https://doi.org/10.17979/spudc.9788497496780.169

DOl libro: https://doi.org/10.17979/spudc.9788497496780

\section{ABSTRACT}

This paper develops the design and implementation of a Service-Learning Project in the subject Organisations Economic Analysis. It is a core subject in the double degree in Business-Law of the University of A Coruna. The aim of applying this methodology is to combine both learning and community service processes while students acquire the competences of the subject previously programmed in the teaching planning. Likewise, this project has been included in Moodle platform with the objective of using information and communication technologies that allow students to develop cooperative learning.

The results show that the Service-Learning Project has allowed students to acquire the competences identified in the teaching planning, both those related to business organisation and management and those related to community engagement. Likewise, both collaborating entity and teachers have given a positive assessment of the development of this project and they have showed their interest in maintaining further collaborations.

KEYWORDS: Service-Learning Project, Information and Communication Technologies, Organisations Economic Analysis. 


\section{INTRODUCCIÓN}

Los cambios sociales, culturales y económicos que se han desarrollado a lo largo de los últimos años, están exigiendo por parte del sistema universitario, no sólo ser formador de profesionales, sino también favorecer el desarrollo de competencias relacionadas con el compromiso cívico y la responsabilidad social. El Espacio Europeo de Educación Superior ha supuesto una oportunidad para establecer nuevas metodologías, tanto activas como pasivas, que ayuden a alcanzar este propósito (Calvo-Bernardino y Mingorance-Arnáiz, 2009; Palomares, 2011). En este contexto, la formación ciudadana es un elemento clave en la educación superior, ya que es difícil ser un buen profesional sin un compromiso ético asociado a cualquier profesión, y sin un compromiso responsable con la sociedad, con su mejora y con el bien común (Herrero, 2006; Santos y Lorenzo, 2012; García et al., 2012).

Por ello la UNESCO (1996) ha planteado que:

(...) la Educación Superior debe reforzar sus funciones de servicio a la sociedad, y más concretamente sus actividades encaminadas a erradicar la pobreza, la intolerancia, la violencia, el analfabetismo, el hambre, el deterioro del medio ambiente y las enfermedades, principalmente mediante un planteamiento interdisciplinario y transdisciplinario para analizar los problemas y las cuestiones planteadas (p. 9)

Tapia (2001) plantea que cuando un servicio comunitario se planifica en la institución educativa en beneficio de fomentar la calidad académica y promover actitudes prosociales en los estudiantes, este servicio comunitario se transforma en un aprendizaje en servicio. Por su parte Roche (1995), plantea que las conductas prosociales pueden ser fortalecidas en proyectos de Aprendizaje-Servicio Comunitario donde se hace énfasis en la enseñanza de valores a través de acciones hacia la comunidad.

Las definiciones de Proyecto de Aprendizaje y Servicio (ApS) tienen en común el establecimiento de una metodología pedagógica que promueve actividades estudiantiles solidarias, no sólo para atender demandas de la sociedad, sino para mejorar la calidad del aprendizaje académico y formar una ciudadanía participativa y solidaria. Se puede notar una 
doble intencionalidad, por un lado el compromiso social y, por otro, el aprendizaje de conocimientos, habilidades, actitudes y valores. De ahí la necesidad de establecer nuevos enfoques de aprendizaje, planificados en beneficio de la comunidad, que es lo que en este trabajo se ha realizado aplicado a la asignatura objeto en cuestión.

\section{DESCRIPCIÓN DE LA EXPERIENCIA}

Dentro de la asignatura Análisis Económico de las Organizaciones, troncal del doble grado Administración y Dirección de Empresas-Derecho de la Universidade da Coruña, se pretende que el alumnado entre en contacto con un colectivo específico. De esta manera, los estudiantes pondrán en práctica los conocimientos aprendidos a lo largo de la asignatura, mediante la realización de informes que respondieron a las problemáticas organizativas de la empresa TRAMEVE (Tratamiento Medioambiental de Vehículos, S.L.), empresa cuyos trabajadores se caracterizan en su mayor parte por tener discapacidad.

Uno de los problemas que se han detectado en la planificación y organización de esta materia es que los casos prácticos que se realizan en el aula no permiten considerar toda la complejidad a la que tienen que hacer frente las empresas reales, ya sea por la incertidumbre del entorno como por la cantidad de variables que les pueden influir de cara a la toma de decisiones. Con la realización de este proyecto se ha podido satisfacer dicha necesidad y los estudiantes han comprobado dicha dificultad. A mayores, el hecho de poder dar un servicio a la comunidad permite satisfacer una necesidad ya detectada por la sociedad que es la falta de empatía con los problemas sociales, así como, la escasez de compromiso por parte de los jóvenes en la mejora de su entorno. Este proyecto, contribuye a despertar en los estudiantes su interés por la acción colectiva, así como su compromiso cívico.

El planteamiento de la experiencia se enmarca plenamente en el Aprendizaje y Servicio puesto que los objetivos son:

- Ofrecer al alumnado la posibilidad de conocer la realidad de una empresa cuyos trabajadores tienen algún tipo de discapacidad, para incorporar dicha información en 
los proyectos que tendrán que realizar como directivos de una organización en su horizonte profesional.

- Facilitar el conocimiento entre un grupo de estudiantes de la Universidade da Coruña y un grupo de trabajadores con discapacidad, colaborando en su proceso de integración en la sociedad.

- Favorecer un marco de reflexión sobre las necesidades de las organizaciones que trabajan con este colectivo, potenciando cambios de actitud y el compromiso hacia colectivos con desventajas sociales.

- Adquirir actitudes favorables hacia un modelo educativo que valora el análisis económico de las organizaciones como un recurso pedagógico de intervención social. La entidad receptora del servicio es la empresa "Tratamiento Medioambiental de Vehículos, S.L." (TRAMEVE). Dicha empresa nace en el año 2000 como consecuencia de un proyecto presentado por la Confederación Gallega de Personas con Discapacidad (COGAMI) al Programa Europeo HORIZON III (1997-1999). El proyecto pretendía disminuir los factores que llevan a las personas con discapacidad física a una situación de exclusión social con la búsqueda de puestos de trabajo relacionados con la recuperación y tratamiento de vehículos fuera de uso.

En cuanto a las personas destinatarias del servicio, nos encontramos, por un lado, al gerente y encargados de la empresa, ya que el alumnado ha de presentar informes en donde planteen posibles soluciones a los problemas organizativos que posee la empresa. Por otro lado, los estudiantes también son destinatarios de este servicio, ya que han podido observar diferentes realidades y situaciones personales, así como problemas reales a los que se enfrentan el colectivo de personas con discapacidad física. A mayores, los estudiantes han aplicado a un caso real los conocimientos teóricos vistos en clase y han desarrollado las siguientes competencias:

- Gestionar y administrar una empresa u organización de pequeño tamaño, entendiendo su ubicación competitiva e institucional e identificando sus fortalezas y debilidades. 
- Emitir informes de asesoramiento sobre situaciones concretas de empresas y mercados.

- Redactar proyectos de gestión global o de áreas funcionales de la empresa.

- Saber aplicar los conocimientos a su trabajo o vocación de una forma profesional por medio de la elaboración y defensa de argumentos y la resolución de problemas dentro de su área de trabajo.

- Desempeñar labores de gestión, asesoramiento y evaluación en las organizaciones empresariales.

- Respetar los derechos fundamentales y de igualdad entre hombres y mujeres, respetar la promoción de los Derechos Humanos y los principios de igualdad de oportunidades, no discriminación y accesibilidad universal de las personas con discapacidad.

- Desarrollarse para el ejercicio de una ciudadanía abierta, culta, crítica, comprometida, democrática y solidaria, capaz de analizar la realidad, diagnosticar problemas, formular e implantar soluciones basadas en el conocimiento y orientadas al bien común.

Por último, también será receptora la sociedad en general, ya que al tratarse de una actividad que integra el servicio a la comunidad, los estudiantes podrán desarrollar un compromiso social que les servirá de base cuando se incorporen al mercado laboral. Dichos valores y actitudes de ayuda a los demás se verán reflejados a lo largo de toda su vida profesional.

Este proyecto de Aprendizaje y Servicio aplicado a la asignatura Análisis Económico de las Organizaciones, ha sido realizado en grupos de dos y tres alumnos, en donde su participación fue voluntaria. La mayor parte del alumnado eligió esta alternativa frente al trabajo tradicional que se realizaba en cursos anteriores. Las razones de dicha elección fueron que consideraban que se trataba de un proyecto más interesante en donde sus posibilidades de aprendizaje iban a ser mayores y que valoraban especialmente del hecho de poder prestar un servicio a la 
sociedad. Los estudiantes que participaron en el ApS fueron 37, de un total de 46, el resto realizó el trabajo tradicional.

Uno de los aspectos más importantes de esta actividad es la implicación del alumnado en todo el proceso de preparación y desarrollo de los informes, donde el compromiso individual de cada uno de los alumnos participantes ha sido esencial para el éxito de este proyecto. A mayores, se ha querido implantar el aprendizaje colaborativo en todo el proceso, empleado el uso de las tecnologías de la información y comunicación (TICs). El trabajo se dividió en tres partes, por un lado, la evolución del sector, seguido de la teoría de los costes de transacción y la teoría de la agencia. Para cada parte del trabajo, se ha creado una wiki en donde cada uno de los componentes de los diferentes grupos ha ido colgando información que consideraba relevante de la empresa para la realización de este proyecto.

Con respecto al sistema de evaluación de la asignatura, los alumnos que participaron en la realización de los informes del Proyecto ApS estaban exentos de realizar uno de los trabajos obligatorios de la asignatura, sustituyéndoles dicha nota por la evaluación que se les daba en dicha actividad. Esta evaluación tenía dos partes diferenciadas: por un lado, la participación en las diferentes wikis y, por otro, la valoración del contenido del trabajo.

En la tabla siguiente se expone el cronograma de las diferentes actividades que se realizaron a lo largo del cuatrimestre: 
Tabla 1. Cronograma de actividades

\begin{tabular}{|l|l|}
\hline Septiembre & $\begin{array}{l}\text { Toma de contacto con la empresa por vía telefónica para explicarles el proyecto y ver si } \\
\text { estarían interesados }\end{array}$ \\
\hline Noviembre & Visita a la empresa en Santiago y entrevista con el gerente \\
\hline Febrero & Selección de estudiantes participantes y formación de grupos de trabajo \\
\hline Marzo & $\begin{array}{l}\text { Presentación de la empresa TRAMEVE y COGAMI (Confederación Gallega de Personas } \\
\text { con Discapacidad) por parte de responsables de las mismas. } \\
\text { Presentación de aspectos relevantes organizativos sobre los cuales trabajar alumnos de } \\
\text { la UDC. }\end{array}$ \\
\hline Abril & $\begin{array}{l}\text { Preparación de materiales para la elaboración de los informes por parte del alumnado de } \\
\text { la UDC. } \\
\text { Desarrollo de wikis colaborativas donde compartir información de la empresa así como } \\
\text { del sector donde desarrolla su actividad. } \\
\text { Desarrollo de informes por parte de cada grupo relacionados con temas de la Teoría de } \\
\text { los Costes de Transacción y Teoría de la Agencia. } \\
\text { Tutorización de los trabajos en grupo. }\end{array}$ \\
\hline Mayo & $\begin{array}{l}\text { Entrega de los informes y evaluación de resultados de la actividad y de las competencias } \\
\text { alcanzadas por los alumnos. } \\
\text { Selección de los mejores informes. }\end{array}$ \\
\hline Junio & $\begin{array}{l}\text { Presentación de los informes por parte de los grupos de trabajo que obtuvieron mayor } \\
\text { nota a la empresa TRAMEVE y a COGAMl. }\end{array}$ \\
\hline
\end{tabular}

\section{RESULTADOS}

El proyecto ApS ha permitido que los alumnos adquirieran las competencias anteriormente descritas, tanto las relacionadas con la organización y gestión de empresas y la toma de decisiones en un ambiente de incertidumbre, como las relacionadas con su compromiso con la comunidad, más concretamente con el problema real de inserción laboral al que se enfrentan las personas con discapacidad y la importancia de que existan este tipo de organizaciones.

Para la evaluación del proyecto se han utilizado dos métodos: Por un lado, los profesores elaboraron una encuesta en donde los estudiantes, una vez participado en el Programa de Aprendizaje y Servicio, valoraban sus posibilidades educativas, ya no sólo en la materia impartida sino en su formación universitaria en general, a mayores se les preguntaba por su 
interés por la acción colectiva. Por otra parte, se les entregó a los estudiantes, al finalizar el proyecto de Aprendizaje y Servicio, una encuesta en papel basada en la encuesta propuesta por Aramburuzabala y García (2012), donde se valoraron los siguientes aspectos: servicio significativo, conexión con el currículum, reflexión, diversidad, compromiso cívico, evaluación del progreso y duración.

A partir de las encuestas realizadas, podemos indicar que todos los agentes implicados en este proyecto (alumnos, empresa y docentes) muestran un alto grado de satisfacción.

En cuanto a los estudiantes universitarios, se caracterizan por haber realizado una valoración positiva de su participación en el proyecto de Aprendizaje y Servicio, tal y como muestra la Figura 1, donde se obtienen valores iguales 0 superiores a 3,4 -en una escala del 1 al 5 , en donde 1 es totalmente en desacuerdo y 5 totalmente de acuerdo- en todos los ítems valorados. Destacando la conexión con el currículum, la comprensión de la diversidad y el ser un servicio significativo.

Figura 1. Valoración de los estudiantes al Proyecto de Aprendizaje y Servicio.

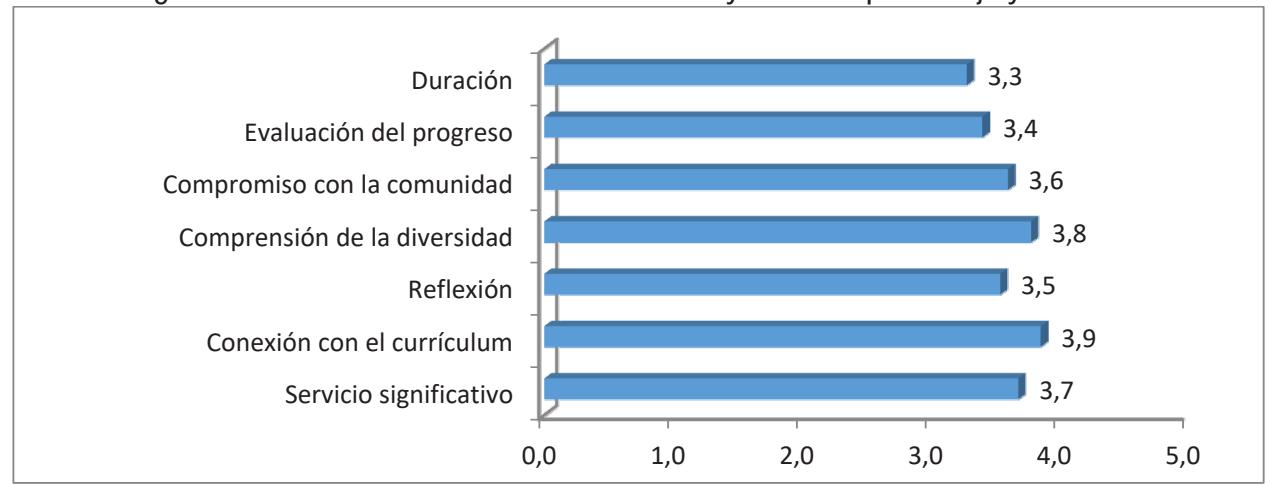

En la Figura 2 se nos muestra la valoración de los estudiantes en cuanto a su compromiso cívico tras la realización del proyecto ApS. Los resultados son muy positivos ya que, en la mayor parte de los casos, los estudiantes han mejorado su motivación para ayudar a los demás y también su sentimiento de poder aportar algo a la sociedad. 
Figura 2. Valoración de los estudiantes sobre su comportamiento una vez realizado el proyecto de Aprendizaje y Servicio.

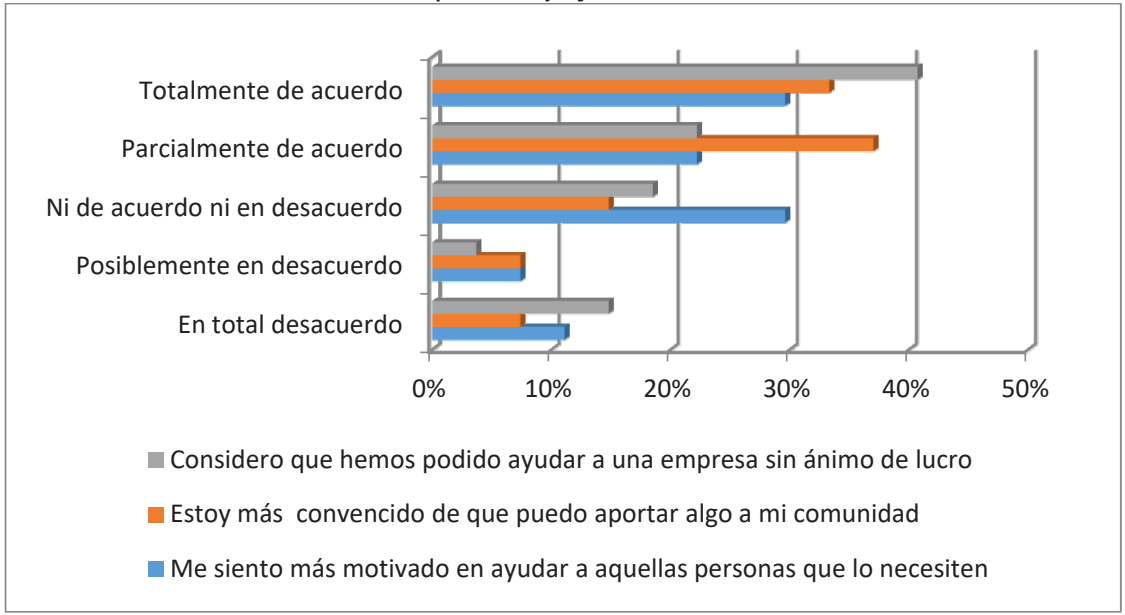

A mayores, se ha querido analizar cuál es la valoración de los estudiantes al uso de Moodle y de wikis colaborativas. Los resultados se muestran en la figura siguiente donde podemos observar la importancia que para los estudiantes tienen las TICs en su formación universitaria, en particular el uso de Moodle. La wiki colaborativa, en contra de nuestra idea inicial, no ha tenido una valoración tan positiva como podríamos haber esperado. La razón principal, desde nuestro entender, está relacionada con la falta de tiempo para realizar el proyecto tal y como estaba previsto, lo que limitó el tiempo de los estudiantes para colgar mayor información en este apartado. 
Figura 3. Valoración de los estudiantes sobre las nuevas tecnologías de información tras la realización del proyecto ApS

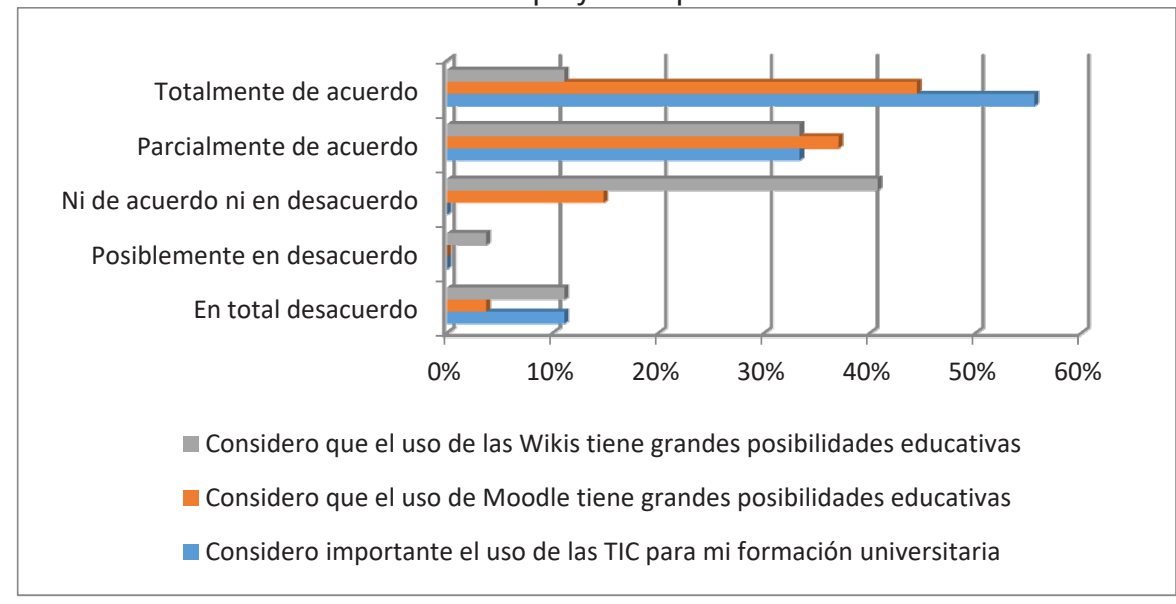

Entre los resultados por parte de la entidad colaboradora y del profesorado, la evaluación que han realizado del presente proyecto de Aprendizaje y Servicio es altamente positiva. Destaca particularmente los planes de acciones colaborativos desarrollados entre la empresa y los profesores para lograr los objetivos establecidos inicialmente en el proyecto. Tanto la entidad colaboradora como los profesores involucrados han mostrado su interés en mantener colaboraciones futuras.

Como cuestiones a mejorar en próximas convocatorias estaría, por un lado, el tiempo, ya que la mayor parte de los alumnos están de acuerdo en que se trata de una actividad lo suficientemente compleja para dedicarle más tiempo del que se había planificado inicialmente. Por otro lado, los alumnos comentan que la empresa en sí no les dio mucho juego para poder aplicar todos los conocimientos aprendidos en clase ya que se trataba de una empresa pequeña con muy poco margen de maniobra. De cara a próximas convocatorias, se tratará de paliar dichas desventajas. 


\section{CONCLUSIONES}

El Aprendizaje y Servicio es un método para unir éxito educativo y compromiso social: aprender a ser competentes siendo útiles a los demás, aprender haciendo un servicio a la comunidad.

Tras la realización de dicho proyecto en la asignatura Análisis Económico de las Organizaciones, las principales conclusiones que podemos extraer son las siguientes:

- Los estudiantes aplicaron sus conocimientos al desarrollo de un informe de asesoramiento sobre cuestiones organizativas a la empresa TRAMEVE.

- Los estudiantes mostraron un mayor interés al tener que aplicar sus conocimientos a una empresa real, donde además ofrecían un servicio a la comunidad (al tratarse de un centro de integración de personas con discapacidad).

- Los estudiantes adquirieron actitudes favorables hacia un modelo educativo que valora el análisis económico de las organizaciones como un recurso pedagógico de intervención social.

- Se promovió el trabajo colaborativo y en grupo mediante el uso de las tecnologías de la información y comunicación.

Reflexiones de los participantes y aspectos a mejorar: Los estudiantes consideran que el presente proyecto de Aprendizaje y Servicio es un servicio significativo, que promueve la comprensión de la diversidad y el respeto mutuo. Si bien, parte de los estudiantes nos han indicado que la duración del mismo no ha sido adecuada y que sería necesario que para posteriores ediciones se prolongase dicha duración en el tiempo.

\section{REFERENCIAS}

- Aramburuzabala, P. \& García, R. (2012). El aprendizaje-servicio en la formación de maestros. Revista del Congrés Internacional de Docència Universitària i Innovació (CIDUI), 1(1) 
- Calvo-Bernardino, A. \& Mingorance-Arnáiz, A. C. (2009). La estrategia de las universidades frente al Espacio Europeo de Educación Superior. Revista Complutense de Educación, 20(2), pp. 319-342.

- García, R., Gozálvez, V., Vázquez, V. \& Escámez, J. (2012). Repensando la Educación: cuestiones y debates para el siglo XXI. Valencia: Brief Ediciones.

- Herrero, M. (2006). Ciudadanía y Universidad. Estudios sobre Educación, 10, pp. 155-173.

- Palomares, A. (2011). El modelo docente universitario y el uso de nuevas metodologías en la enseñanza, aprendizaje y evaluación. Revista de Educación, 355, pp. 591-604.

- Roche, R. (1995). Psicología y educación para la prosocialidad. Barcelona: Colección Ciencia y Técnica- Universidad Autónoma de Barcelona.

- Santos, M. A. \& Lorenzo, M. (2012). Dimensión cívica y desarrollo formativo de los estudiantes universitarios en el contexto español. Revista Electrónica de Investigación Educativa Número Especial. Recuperado de: http://redie.uabc.mx/NumEsp2/contenido-rego.html

- Tapia, M.N. (2008). Aprendizaje y servicio solidario. Buenos Aires: Ciudad Nueva.

- UNESCO (1996). La educación encierra un tesoro: informe de la Unesco. Comisión Internacional sobre la Educación para el Siglo XXI. Madrid: Santillana/Unesco. 